\title{
Blood Pressure Variability: Assessment, Prognostic Significance, and Management
}

\author{
Meenakshi Chenniappan
}

\begin{abstract}
Over the years, physicians and researches alike have tried to understand the parameter in blood pressure which would offer as an ideal risk marker to predict cardiovascular events. Our focus has shifted from diastolic pressure to systolic pressure to pulse pressure, etc. In spite of optimal control of all the above target pressures, hypertensive subjects still end up with stroke and high blood pressure has been noted as a defining factor in them. This has led to physicians identifying a phenomenon called blood pressure variability. Individuals exhibiting similar clinic or home blood pressure can differ considerably with respect to their average day and night time values, beat-bybeat blood pressure variation during wakefulness and sleep, responses to mental and physical stimuli, and intersession and seasonal variation. There is now evidence that several such representations of blood pressure variability, if augmented, increase cardiovascular risk independent of the average of conventionally acquired blood pressure readings.
\end{abstract}

Keywords: Antihypertensive drugs, $\beta$-blockers, Placebo.

How to cite this article: Chenniappan M. Blood Pressure Variability: Assessment, Prognostic Significance, and Management. Hypertens J 2016;2(3):124-130.

\section{Source of support: Nil}

Conflict of interest: None

\section{INTRODUCTION}

Interest in blood pressure variability (BPV) as a cardiovascular $(\mathrm{CV})$ risk factor has gained focus in recent times due to evidence which suggests that increased BPV places added strain on the CV system unrelated to its average value, leading to increased risk of target organ damage (TOD) and CV events. ${ }^{1,2}$ Blood pressure values undergo spontaneous variations due to various reasons. Short-term variations may occur within the 24 hours due to day-night changes and also among hours, minutes, and even adjacent beats. Long-term variations may occur due to differences among days, months, and seasons. Additionally, systolic BP (SBP) increases with age

\footnotetext{
Senior Consultant

Department of Cardiology, Ramakrishna Nursing Home and Apollo Speciality Hospital, Chennai, Tamil Nadu, India

Corresponding Author: Meenakshi Chenniappan, Senior Consultant, Department of Cardiology, Ramakrishna Nursing Home and Apollo Speciality Hospital, Trichy, Chennai, Tamil Nadu, India e-mail: chennidr@gmail.com
}

and diastolic BP (DBP) exhibits an age-related biphasic change. ${ }^{3}$ Treatment with antihypertensive medications might help in reducing BPV, in addition to obtaining mean BP control, conferring optimal CV protection. To this end, understanding the basics of BPV might prove valuable.

\section{TYPES OF BPV AND MECHANISMS}

\section{Very Short-term BPV}

The beat-to-beat changes in $\mathrm{BP}$ occur due to interaction between several CV regulatory systems, such as the baroreceptor reflex, the renin-angiotensin system, the vascular myogenic response, and the release of nitric oxide from the endothelium. ${ }^{4}$ The identification of these BPV influencers can be done by power spectral analysis and may provide important information on individual BP control mechanisms. ${ }^{5}$ Very short-term BPV or beat-to-beat variability is measured by noninvasive continuous monitoring of $\mathrm{BP}$ through finger cuffs equipped with an infrared photoplethysmograph and technology-based quantification of finger blood pressure levels. Several newer noninvasive devices are also being developed. ${ }^{6}$ However, since most recordings are done under laboratory settings, its reliability outside of the controlled environment is suspect. ${ }^{7}$

\section{Short-term or 24-hour BPV}

Twenty-four-hour BPV occurs due to several factors, such as physical activity, sleep, and emotional stimuli. Day and night changes may be influenced by signals initiated by the brain. Blood pressure variability also occurs due to mechanical forces generated by ventilation and due to humoral and local vasomotor phenomena. These BP changes are regulated largely via the baroreflex and also to some extent through the sympathetic nervous system and certain nonneural mediators (Fig. 1). ${ }^{3}$

Nighttime BP (sleep) is on an average 10 to $20 \%$ lower than that during daytime (waking hours). However, in hypertensive patients, the 24-hour BPV patterns might be diverse. Some show $>20 \%$ or $<10 \%$ decrease in BP at night, and some may even show a rise in nighttime $\mathrm{BP}$ as compared with daytime values. Depending on their SBP values, these patients are usually categorized as extreme dippers (night-day BP ratio $\leq 0.8)$, dippers $(0.8<$ ratio $\leq 0.9$ ), nondippers $(0.9<$ ratio $\leq 1.0)$, and reverse dippers 
Blood Pressure Variability: Assessment, Prognostic Significance, and Management

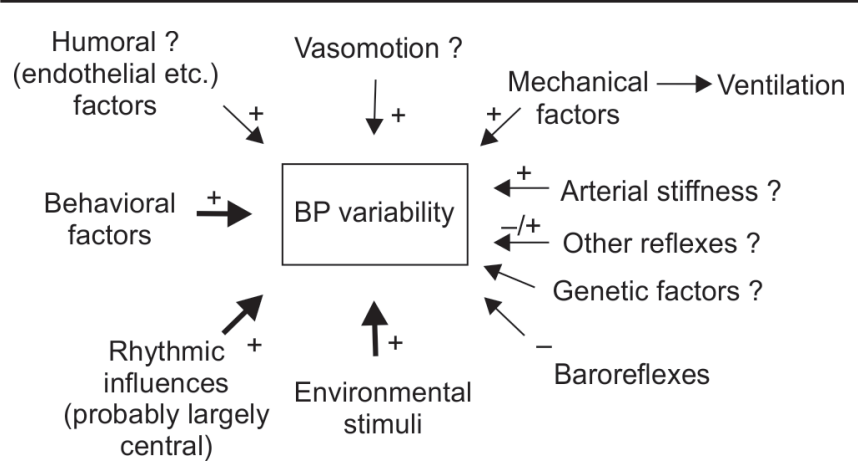

Fig. 1: Factors involved in 24-hour BP variability. The sign "+" refers to factors favoring and the sign "-" to factors opposing BP variations. The query indicates absence of conclusive evidence. Source: Mancia $^{3}$

or risers (ratio $>1.0$ ). Reverse dippers seem to present with worse outcomes than dippers, and the proposed reasons for this $\mathrm{BP}$ rise at night include nocturnal autonomic dysfunction, disturbed baroreflex sensitivity, sleep apnea, abnormal sodium handling, and nocturnal volume overload. ${ }^{8}$

\section{Long-term BPV}

Long-term BPV includes day-to-day, visit-to-visit (VVV), and seasonal variations. Behavioral changes play a major role in long-term BPV as suggested by the clear differences observed in ambulatory BP (ABP) values during weekdays and weekends. Inadequate BP treatment due to poor treatment compliance by the patient or improper dosing/titration of the antihypertensive medication by the physician might also influence long-term BPV. Blood pressure measurement errors play a major role in VVV BPVs. Lastly, BP levels vary between summer and winter, indicating the influence of temperature and daylight hours brought about by seasonal changes. ${ }^{9}$ Data from the Multiethnic Study of Atherosclerosis (MESA) showed that arterial stiffness may also contribute to long-term BPV. Lower arterial elasticity and reduced aortic distensibility were observed in patients with higher VVV BPV in this study. ${ }^{10}$

\section{METHODS OF BPV MEASUREMENT}

The various methods of obtaining BPV measurements include continuous beat-to-beat $\mathrm{BP}$ recordings, repeated office BP measurement (OBPM), 24-hour ambulatory BP monitoring (ABPM), or home BP monitoring (HBPM) over long periods of time. ${ }^{7}$

The key index of short-term BPV is standard deviation (SD) of the 24-hour average ABP values. Since nighttime fall in BP might interfere with accurate BPV assessment, it has been proposed that 24-hour SD can be alternatively expressed as the weighted mean of daytime and nighttime values. $^{11}$ The average SD of BP can also be divided by the corresponding mean BP and multiplied by 100 to express a normalized measure of BPV as a coefficient of variation $(\mathrm{CoV}){ }^{7}$ Another $\mathrm{BPV}$ index is average real variability or ARV. It denotes the average of the absolute differences between consecutive BP measurements and has been found to be a more reliable prognostic indicator than SD. ${ }^{7,12}$

Day-to-day BPV can be assessed by ABPM for over 48 hours, which may not be tolerated by all patients. The alternative is use of HBPM that can accumulate data over several days. Availability of day-to-day BPV data might help the physician streamline the hypertensive treatment earlier as compared with VVV BP measurement. ${ }^{7}$

Visit-to-visit BPV can be assessed by ABPM or OBPM. However, OBPM is an imperfect tool to assess VVV BPV since it might not reflect the BP burden during the patient's normal activities and requires consistent visits to the physician's office. Since ABPM cannot be repeated frequently, it can be an inefficient tool to measure VVV BPV. On the other hand, HBPM seems ideally suited for this assessment without the presence of the "white coat effect" and can be obtained under fairly constant conditions. ${ }^{7}$

Table 1 offers a quick review of the various parameters associated with BPV.

\section{BLOOD PRESSURE VARIABILITY: A NOVEL PREDICTOR OF TOD AND CV EVENTS ACROSS PATIENT SUBGROUPS}

The prognostic importance of both short- and long-term BPV as outlined in some key studies are discussed below.

\section{Short-term BPV}

Mancia et $\mathrm{al}^{13}$ conducted one of the earliest studies where the relationship between 24-hour BPV and BP mean was assessed by intra-arterial BP measurement over 24 hours. Results showed that BPV (SD of the 24 hours, day and night mean BPs) increased in patients with hypertension compared with normotensive individuals. The degree of BPV was generally linked to the mean BP values. It follows that reduction of mean BP by antihypertensive medications also results in BPV reduction. ${ }^{3}$

Several studies have established the impact of shortterm BPV on TOD and CV events in hypertensive patients. Parati et $\mathrm{al}^{14}$ studied 108 hospitalized subjects with mildto-severe hypertension and found that lower 24-hour BPV was associated with significantly lower prevalence and severity of TOD compared with those with higher 24-hour BPV ( $p<0.05)$. Cardiac structural and vascular alterations have also been attributed to 24-hour BPV, making it an independent predictor for CV events. A study conducted on 73 hypertensive patients followed up for an average of 7.4 years showed that the severity of 

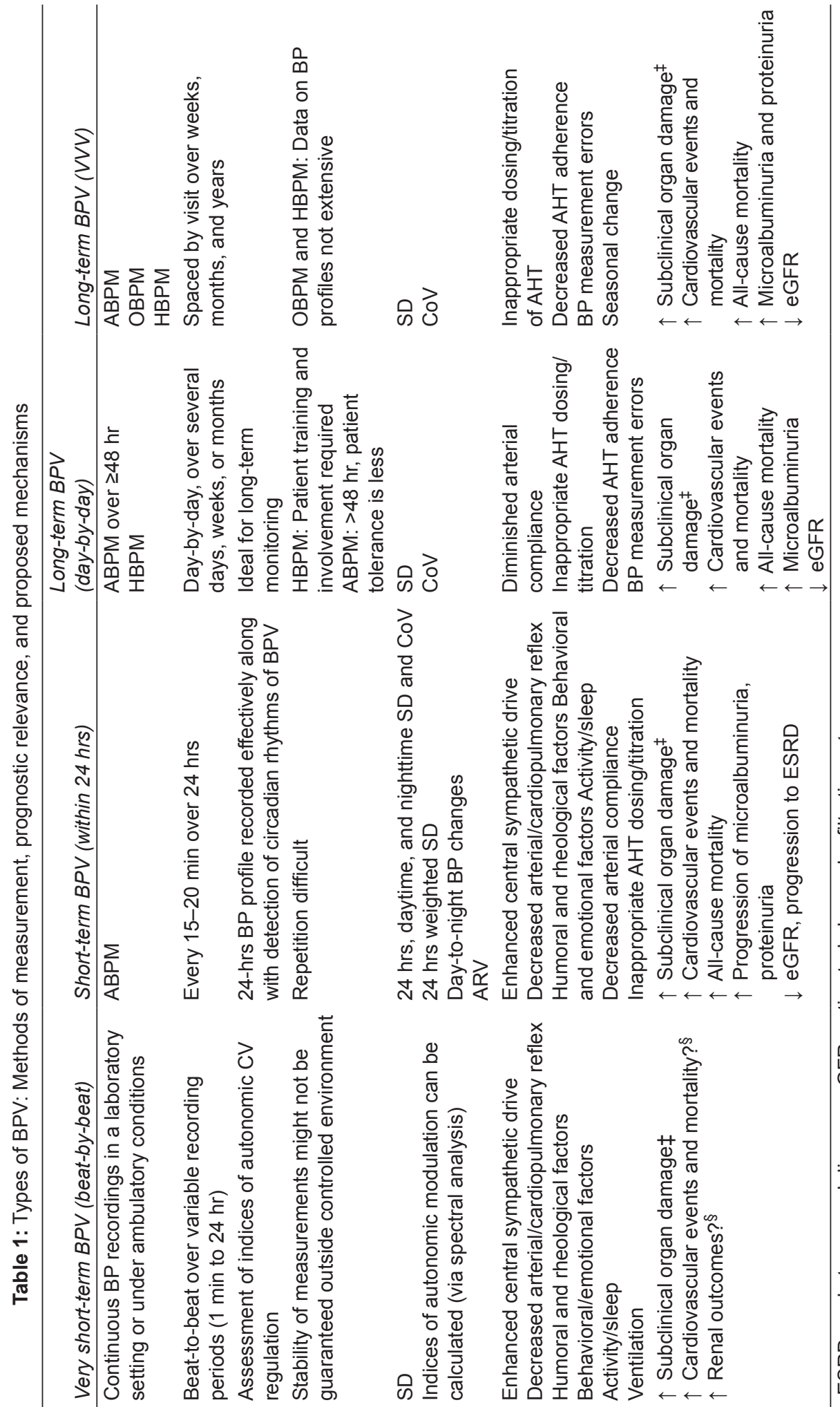

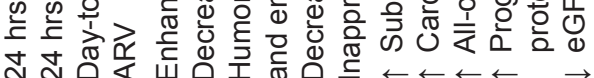
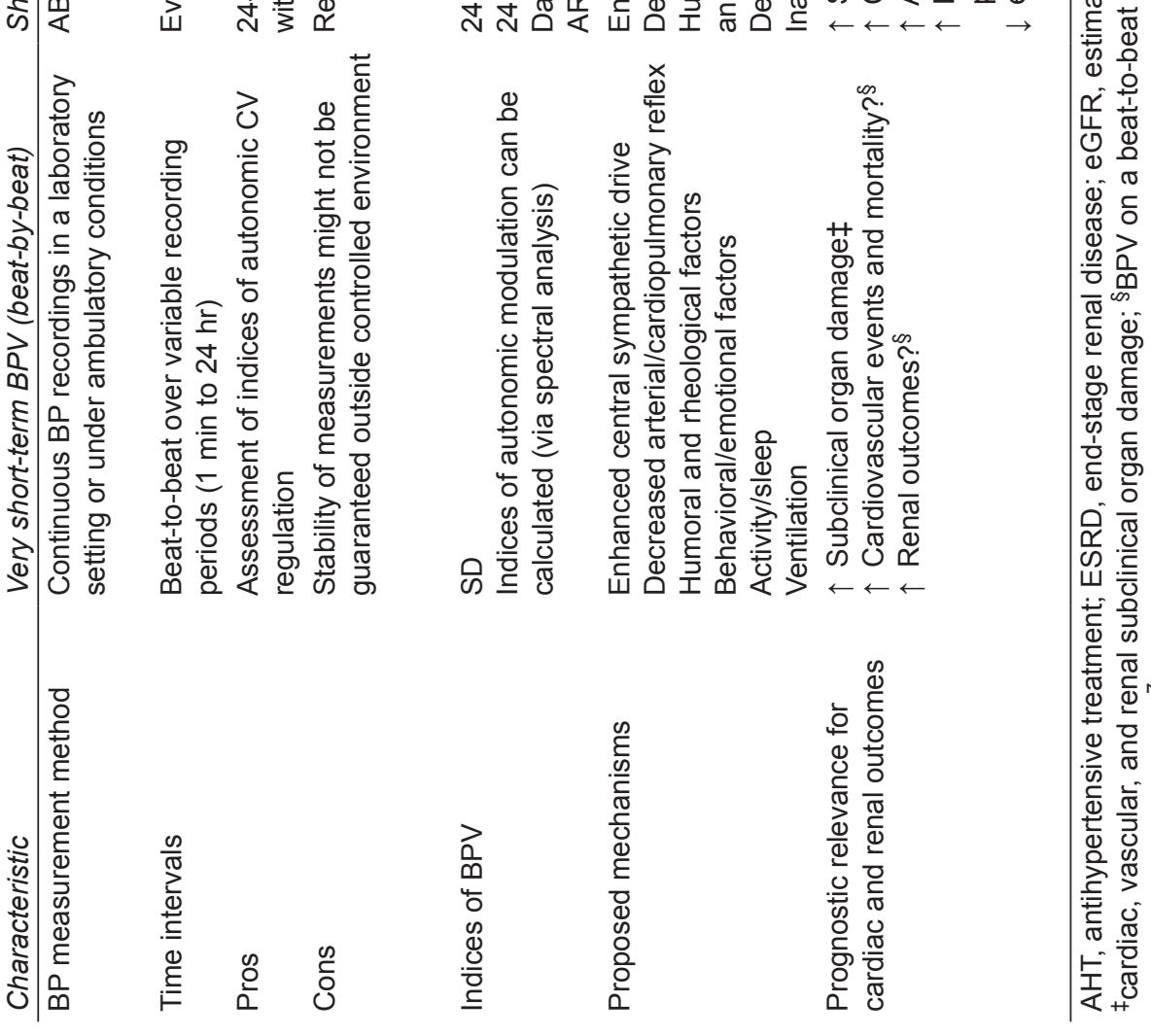

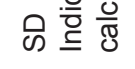
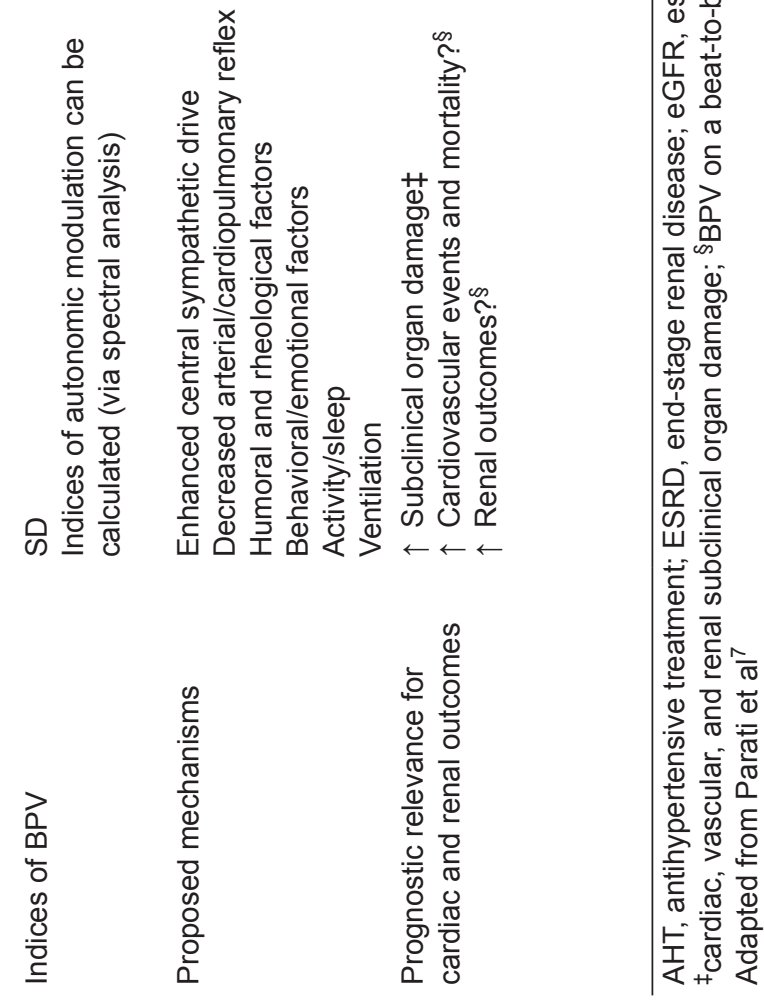


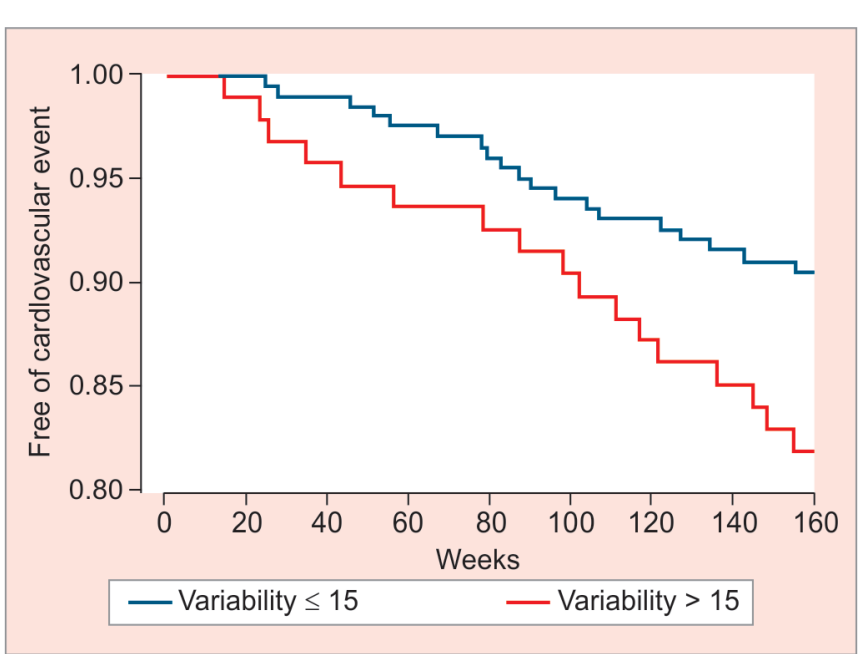

Graph 1: Kaplan-Meier survival analysis for fatal and nonfatal CV morbid events in patients with increased $(>15 \mathrm{~mm} \mathrm{Hg}$ ) and normal $(\leq 15 \mathrm{~mm} \mathrm{Hg})$ BP variability. Survival analysis adjusted for IHD. Source: Sander et al ${ }^{16}$

TOD, especially increase in left ventricular mass index, was related to baseline 24-hour BPV. Cardiovascular complications of hypertension may thus depend on the degree of 24-hour BPV. ${ }^{15}$

In a study by Sander et al ${ }_{1}^{16}$ the progression of carotid intima-media thickness, a marker for atherosclerosis, was significantly greater in patients with increased systolic BPV (SBPV) during 3.3 years of follow-up. Kaplan-Meier survival analysis (Graph 1) showed a significantly higher rate of $\mathrm{CV}$ morbid events with increased BPV. Consequently, the relative risk of $\mathrm{CV}$ events was also significantly increased in these patients, even after adjusting for other risk factors.

The Pressioni Arteriose Monitorate e Loro Associazioni (PAMELA) Study evaluated the ability of 24-hour $\mathrm{BPV}$ to predict long-term risk of death over a period of 12 years. Results showed that CV mortality was inversely correlated with the day/night BP difference. Conversely, higher residual BP variability [day and night erratic DBP changes detected by eliminating the cyclic components (nocturnal and postprandial BP falls) assessed via Fourier spectral analysis] resulted in higher risk for fatal $\mathrm{CV}$ events. This study reaffirmed that BPV, especially erratic $\mathrm{BPV}$, has prognostic value in hypertension over and above that of mean BP values. ${ }^{17}$

Diurnal changes in BP have also been shown to be predictors of CV risk in hypertensive patients with/ without $\mathrm{CV}$ disease at baseline. A meta-analysis conducted on the significance of the nighttime $\mathrm{ABP}$ in 3,468 hypertensive patients without history of major CV disease showed that reverse dippers were at higher risk for $\mathrm{CV}$ disease than dippers. Also, all-cause mortality was found to be lower in extreme dippers than in dippers, independent of mean 24-hour BP values and other variables. In another analysis conducted among 302 patients with CV disease at baseline, significantly increased all-cause mortality and incidence of $C V$ events was observed in reverse dippers compared with dippers, both before and after adjustment for 24-hour BP values. ${ }^{8}$

\section{Long-term BPV}

The prognostic value of variability in day-by-day HBPM was assessed by Kikuya et $\mathrm{al}^{18}$ among 2,455 residents of Ohasama, Japan. Over a follow-up period of 12 years, day-to-day BP and heart rate (HR) variability (measured as within-subject SDs of home measurements) were predictors of $\mathrm{CV}$ mortality, after adjusting for several risk factors. Standard deviations of SBP and HR were significantly and independently linked to CV mortality. Specifically, SD of SBP foretold risk of stroke and cerebral infarction.

In the Finn-Home study, Johansson et a ${ }^{19}$ evaluated the prognostic value of BPV via HBPM and HR measured for seven consecutive days in a sample of 1,866 Finnish adults. After a follow-up of 7.8 years, higher variability of morning-evening and morning day-by-day home BP, and corresponding $\mathrm{HR}$ values predicted $\mathrm{CV}$ events after adjusting for several relevant risk factors.

Results from the Anglo-Scandinavian Cardiac Outcomes Trial - Blood Pressure Lowering Arm (ASCOTBPLA) trial also showed that patients with good control of mean BP but greater residual SBPV had a five times higher risk of stroke than those with lower variability values, establishing VVV BPV as a key predictor of the long-term risk of stroke after transient ischemic attack. ${ }^{20}$

Data from the National Health and Nutrition Examination Survey (NHANES) III conducted on 956 subjects demonstrated that SD of VVV SBPV $>4.80$ $\mathrm{mm} \mathrm{Hg}$ was associated with a $57 \%$ increase in overall mortality. ${ }^{21}$ Another meta-analysis involving 77,299 patients over 6.3 years showed that VVV SBPV can predict all-cause and CV mortality and stroke, independent of age and mean SBP. ${ }^{22}$

The impact of long-term BPV on mortality risk was assessed in a study by Hastie et al. ${ }^{23}$ A total of 522 hypertensive patients were followed up for four periods of time after the first visit: Year 1, years 2 to 5 , years 5 to 10 , and years $>10$. Results showed that both SBPV and DBPV for the 1 to 4 year and 5 to 9 years time frame were predictive of mortality irrespective of the mean $\mathrm{BP}$ values. Moreover, this association held true for all-cause, $\mathrm{CV}$, and non-CV mortality.

Data from 58,228 postmenopausal women involved in the Women's Health Initiative followed up for 5.4 years showed that higher annual VVV SBPV was correlated with an increased stroke risk after adjusting for several factors. $^{24}$ 
In a study conducted on 2,161 type 2 diabetic patients over 5.5 years, VVV BPV significantly predicted all-cause mortality, irrespective of mean BP values. ${ }^{25}$ Ushigome et $\mathrm{al}^{26}$ evaluated the correlation between dayby-day variability in HBP on 14 consecutive days and macroalbuminuria in 858 Japanese patients with type 2 diabetes. They found that CoVs of morning and evening SBP were significantly higher in macroalbuminuric patients compared with those without macroalbuminuria. After adjusting for several factors, further analyses showed that CoVs of morning SBP and DBP as well as those of evening SBP were independently linked with the logarithm of urinary albumin excretion (UAE). Coefficient of variation of HBP was thus a novel factor correlated with macroalbuminuria, after accounting for known risk factors in patients with type 2 diabetes.

Patients on hemodialysis with left ventricular hypertrophy formed the treatment population in the Fosinopril in Dialysis (FOSIDIAL) study, which evaluated the safety and efficacy of long-term fosinopril treatment vs placebo on CV clinical outcomes. Further analyses of data on 388 FOSIDIAL patients showed that VVV SBP and DBPV were strongly predictive of $C V$ events in this group. In fact, event-free survival was lowest in the patients with highest CoV SBP (Graph 2). ${ }^{27}$

An analysis of chronic kidney disease subjects from the African American Study of Kidney Disease and Hypertension (AASK) trial showed that VVV SBPV was associated with increased overall and CV mortality. ${ }^{28}$

\section{BLOOD PRESSURE VARIABILITY MANAGEMENT: PROTECTION OFFERED BY ANTIHYPERTENSIVE DRUGS}

Since both short-term and long-term BPV contribute to TOD and CV events in patients with hypertension or diabetes, appropriate antihypertensive treatment that

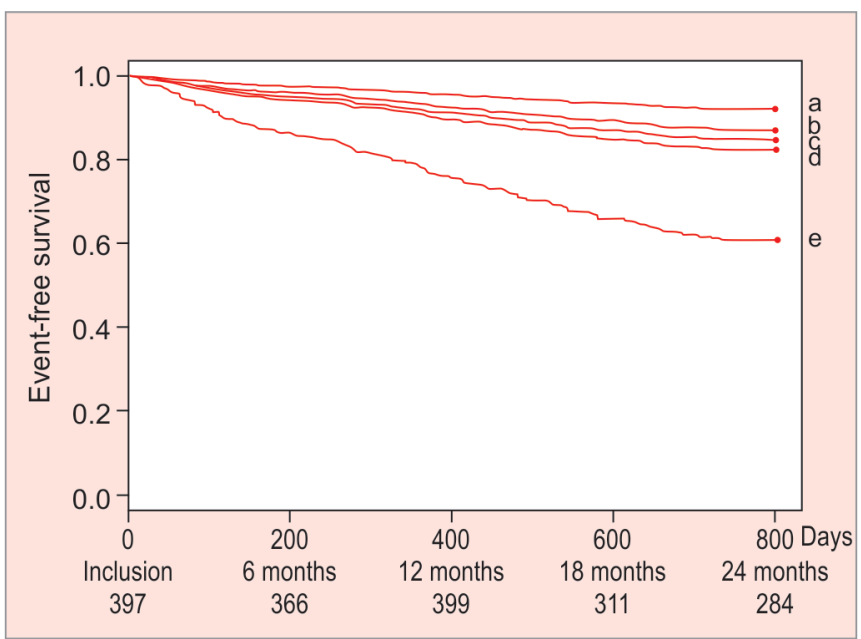

Graph 2: Cox prediction of CV events reduces this variability, in addition to reducing mean $\mathrm{BP}$, might prove to be a beneficial target for $\mathrm{CV}$ prevention. Recent data suggest that there is interdrug variation in efficacy, with calcium channel blockers (CCBs), such as amlodipine proving superior to other drugs in reducing $\mathrm{BPV}^{29}$

A post hoc analysis of the ASCOT-BPLA and the Medical Research Council (MRC) trial was conducted to study the differential impact of $\beta$-blockers and CCBs on BPV and stroke risk. ASCOT-BPLA compared amlodipine-based regimens with atenolol-based regimens in 19,257 patients with hypertension and other vascular risk factors, while the MRC trial compared atenolol-based and diuretic-based regimens vs placebo in 4,396 hypertensive patients. ${ }^{20}$

In ASCOT-BPLA, within-visit, VVV, and ABPM BPV were all reduced by amlodipine-based treatment, irrespective of its effect on mean BP, whereas BPV increased with atenolol-based regimen. The reduced event rate with amlodipine-based regimen was explained by its beneficial effect on VVV BPV. In the MRC trial, SBPV was increased in the atenolol group compared with placebo as well as diuretic group, and stroke risk was allied with the effect on BPV. Optimal prevention of stroke would thus occur with a drug that effectively reduces mean BP without increasing BPV, ideally reducing both.

A meta-analysis of 389 trials showed that there were differential effects of antihypertensive drugs on BPV. Calcium channel blockers and nonloop diuretic drugs reduced interindividual SBPV, whereas angiotensinconverting enzyme inhibitors (ACEIs), angiotensin-2 receptor blockers (ARBs), and $\beta$-blockers increased it, with CCBs showing maximal effect vs placebo (Graph 3). This aspect might be the key reason for the differences in effects of antihypertensive drugs on stroke risk. ${ }^{30}$ Addition of CCBs to other antihypertensive agents

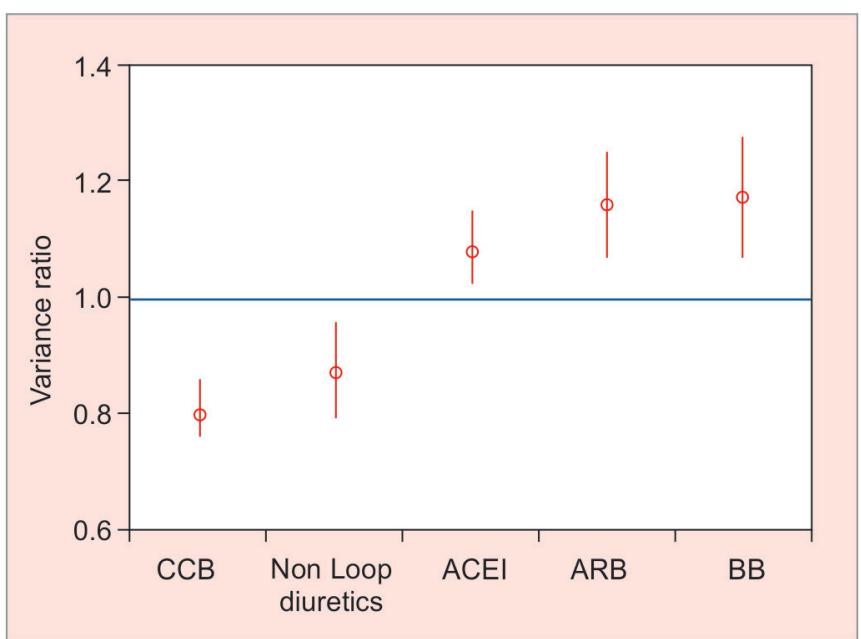

Graph 3: Interindividual variation in SBP compared with other drugs 


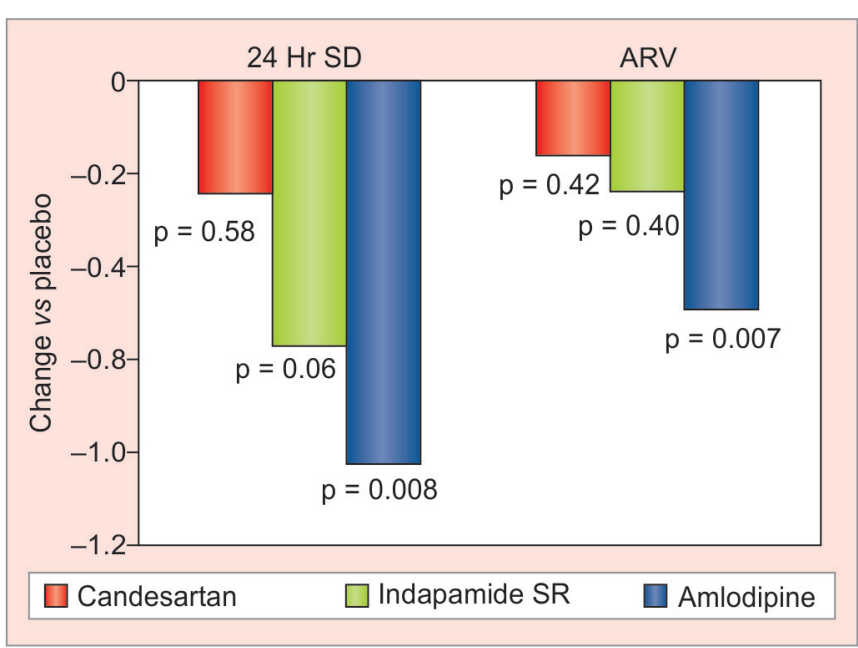

Graph 4: Changes in SBP and its variability by treatment group vs placebo after 3-month treatment

significantly reduced VVV BPV; however, the reverse was not true. ${ }^{4}$

The influence of antihypertensive treatment with candesartan, indapamide sustained release, or amlodipine on SD of 24-hour ABPM in 577 patients was assessed in the NatriliX SR versus CandEsartan and amLodipine in the reduction of systoLic blood prEssure in hyperteNsive patienTs (X-CELLENT) study. After 3 months, amlodipine significantly decreased daytime, nighttime, and 24-hours SBPV, whereas indapamide sustained release significantly decreased SBPV in the daytime and after 24 hours. Conversely, candesartan did not reduce SBPV significantly in any time frame (Graph 4). Even after adjustment for mean BP reduction, only amlodipine was efficacious across all time frames. ${ }^{4,31}$

A secondary analysis of Antihypertensive and Lipid-Lowering Treatment to Prevent Heart Attack Trial (ALLHAT) calculated the effect of amlodipine, chlorthalidone, and lisinopril on VVV BPV across a period of 6 to 28 months. Results showed that even after adjusting for mean BP, amlodipine and chlorthalidone reduced VVV $\mathrm{BPV}$ to a greater extent than lisinopril. ${ }^{32}$

Combination of antihypertensive drugs has also proven effective in reducing BPV. In a study by Hoshino et $\mathrm{al}^{33}$ on 31 hypertensive patients, the combination of amlodipine and olmesartan given at bedtime reduced the morning BP surge effectively, corrected the nocturnal BP fall, and improved UAE.

In another study by Levi-Marpillat et $\mathrm{l}^{34}$ the efficacy of monotherapy and combination therapy on shortterm BPV was assessed on 2,780 hypertensive patients. Calcium channel blockers, followed by diuretics, were correlated with lower short-term BPV compared with ARBs, ACEIs, and $\beta$-blockers. Also, the combination of CCBs and diuretics resulted in lowest short-term BPV compared with other combinations.

\section{CONCLUSION}

The impact of BPV on TOD and CV events across hypertensive, diabetic, and kidney disease patients is well established. Recent data suggest that some antihypertensive drugs are capable of reducing BPV in addition to their efficacy in reducing mean BP values. Among the available antihypertensive agents, CCBs, such as amlodipine have been found to be most effective in reducing BPV, either as monotherapy or in combination with other molecules. Use of amlodipine and its combinations to attenuate $\mathrm{BPV}$ might be a step in the right direction to prevent $\mathrm{CV}$ morbidity and mortality.

\section{REFERENCES}

1. Mancia G, Fagard R, Narkiewicz K, Redon J, Zanchetti A, Böhm M, Christiaens T, Cifkova R, De Backer G, Dominiczak A, et al. 2013 ESH/ESC Guidelines for the management of arterial hypertension: the Task Force for the Management of Arterial Hypertension of the European Society of Hypertension (ESH) and of the European Society of Cardiology (ESC). Eur Heart J 2013 Jul;34(28):2159-2219.

2. Stergiou GS, Ntineri A, Kollias A, Ohkubo T, Imai Y, Parati G. Blood pressure variability assessed by home measurements: a systematic review. Hypertens Res 2014 Jun;37(6):565-572.

3. Mancia G. Short- and long-term blood pressure variability: present and future. Hypertension 2012 Aug;60(2):512-517.

4. Höcht C. Blood pressure variability: prognostic value and therapeutic implications. ISRN Hypertens 2013(Article ID 398485):1-16.

5. Stauss HM. Identification of blood pressure control mechanisms by power spectral analysis. Clin Exp Pharmacol Physiol 2007 Apr; 34(4):362-368.

6. ParatiG,OngaroG, BiloG,Glavina F, Castiglioni P,DiRienzoM, Mancia G. Non-invasive beat-to-beat blood pressure monitoring: new developments. Blood Press Monit 2003;8(1):31-36.

7. Parati G, Ochoa JE, Lombardi C, Bilo G. Assessment and management of blood-pressure variability. Nat Rev Cardiol 2013 Mar;10(3):143-155.

8. Fagard RH. Dipping pattern of nocturnal blood pressure in patients with hypertension. Expert Rev Cardiovasc Ther 2009 Jun;7(6):599-605.

9. Parati G, Liu X, Ochoa JE, Bilo G. Prognostic relevance of blood pressure variability: role of long-term and very long-term blood pressure changes. Hypertension 2013 Oct;62(4):682-684.

10. Shimbo D, Shea S,McClelland RL, Viera AJ, Mann D, Newman J, Lima J, Polak JF, Psaty BM, Muntner P. Associations of aortic distensibility and arterial elasticity with long-term visitto-visit blood pressure variability: The Multi-Ethnic Study of Atherosclerosis (MESA). Am J Hypertens 2013 Jul;26(7): 896-902.

11. Bilo G, Giglio A, Styczkiewicz K, Caldara G, Kawecka-Jaszcz K, Mancia G, Parati G. How to improve the assessment of 24-h blood pressure variability. Blood Press Monit 2005 Dec;10(6):321-323.

12. Mena L, Pintos S, Queipo NV, Aizpúrua JA, Maestre G, Sulbarán T. A reliable index for the prognostic significance of blood pressure variability. J Hypertens 2005 Mar;23(3): 505-511. 
13. Mancia G, Ferrari A, Gregorini L, Parati G, Pomidossi G, Bertinieri G, Grassi G, di Rienzo M, Pedotti A, Zanchetti A. Blood pressure and heart rate variabilities in normotensive and hypertensive human beings. Circ Res 1983 Jul;53(1): 96-104.

14. Parati G, Pomidossi G, Albini F, Malaspina D, Mancia G. Relationship of 24-hour blood pressure mean and variability to severity of target-organ damage in hypertension. J Hypertens 1987 Feb;5(1):93-98.

15. Frattola A, Parati G, Cuspidi C, Albini F, Mancia G. Prognostic value of 24-hour blood pressure variability. J Hypertens 1993 Oct;11(10):1133-1137.

16. Sander D, Kukla C, Klingelhöfer J, Winbeck K, Conrad B. Relationship between circadian blood pressure patterns and progression of early carotid atherosclerosis: a 3-year follow-up study. Circulation 2000 Sep;102(13):1536-1541.

17. Mancia G, Bombelli M, Facchetti R, Madotto F, Corrao G, Trevano FQ, Grassi G, Sega R. Long-term prognostic value of blood pressure variability in the general population: results of the Pressioni Arteriose Monitorate e Loro Associazioni Study. Hypertension 2007 Jun;49(6):1265-1270.

18. Kikuya M, Ohkubo T, Metoki H, Asayama K, Hara A, Obara T, Inoue R, Hoshi H, Hashimoto J, Totsune K, et al. Day-by-day variability of blood pressure and heart rate at home as a novel predictor of prognosis: the Ohasama study. Hypertension 2008 Dec;52(6):1045-1050.

19. Johansson JK, Niiranen TJ, Puukka PJ, Jula AM. Prognostic value of the variability in home-measured blood pressure and heart rate: the Finn-Home Study. Hypertension 2012 Feb;59(2):212-218.

20. Rothwell PM, Howard SC, Dolan E, O'Brien E, Dobson JE, Dahlöf B, Poulter NR, Sever PS; ASCOT-BPLA and MRC Trial Investigators. Effects of beta blockers and calcium-channel blockers on within-individual variability in blood pressure and risk of stroke. Lancet Neurol 2010 May;9(5):469-480.

21. Muntner P, Shimbo D, Tonelli M, Reynolds K, Arnett DK, Oparil S. The relationship between visit-to-visit variability in systolic blood pressure and all-cause mortality in the general population: findings from NHANES III, 1988 to 1994. Hypertension 2011 Feb;57(2):160-166.

22. Tai C, Sun Y, Dai N, Xu D, Chen W, Wang J, Protogerou A, van Sloten TT, Blacher J, Safar ME, et al. Prognostic significance of visit-to-visit systolic blood pressure variability: a metaanalysis of 77,299 patients. J Clin Hypertens (Greenwich) 2015 Feb;17(2):107-115.

23. Hastie CE, Jeemon $\mathrm{P}$, Coleman $\mathrm{H}$, McCallum L, Patel $\mathrm{R}$, Dawson J, Sloan W, Meredith P, Jones GC, Muir S, et al. Longterm and ultra long-term blood pressure variability during follow-up and mortality in 14,522 patients with hypertension. Hypertension 2013 Oct;62(4):698-705.

24. Shimbo D, Newman JD, Aragaki AK, LaMonte MJ, Bavry AA, Allison M, Manson JE, Wassertheil-Smoller S. Association between annual visit-to-visit blood pressure variability and stroke in postmenopausal women: data from the women's health initiative. Hypertension 2012 Sep;60(3):625-630.

25. Hsieh YT, Tu ST, Cho TJ, Chang SJ, Chen JF, Hsieh MC. Visitto-visit variability in blood pressure strongly predicts allcause mortality in patients with type 2 diabetes: a 5.5-year prospective analysis. Eur J Clin Invest 2012 Mar;42(3):245-253.

26. Ushigome E, Fukui M, Hamaguchi M, Senmaru T, Sakabe K, Tanaka M, Yamazaki M, Hasegawa G, Nakamura N. The coefficient variation of home blood pressure is a novel factor associated with macroalbuminuria in type 2 diabetes mellitus. Hypertens Res 2011 Dec;34(12):1271-1275.

27. Rossignol P, Cridlig J, Lehert P, Kessler M, Zannad F. Visitto-visit blood pressure variability is a strong predictor of cardiovascular events in hemodialysis: insights from FOSIDIAL. Hypertension 2012 Aug;60(2):339-346.

28. McMullan CJ, Bakris GL, Phillips RA, Forman JP. Association of BP variability with mortality among African Americans with CKD. Clin J Am Soc Nephrol 2013 May;8(5):731-738.

29. Dolan E, O'Brien E. Blood pressure variability: clarity for clinical practice. Hypertension 2010 Aug;56(2):179-181.

30. Webb AJ, Fischer U, Mehta Z, Rothwell PM. Effects of antihypertensive-drug class on interindividual variation in blood pressure and risk of stroke: a systematic review and meta-analysis. Lancet 2010 Mar;375(9718):906-915.

31. Zhang Y, Agnoletti D, Safar ME, Blacher J. Effect of antihypertensive agents on blood pressure variability: the natrilix SR versus candesartan and amlodipine in the reduction of systolic blood pressure in hypertensive patients (X-CELLENT) study. Hypertension 2011 Aug;58(2):155-160.

32. Muntner P, Levitan EB, Lynch AI, Simpson LM, Whittle J, Davis BR, Kostis JB, Whelton PK, Oparil S. Effect of chlorthalidone, amlodipine, and lisinopril on visit-to-visit variability of blood pressure: results from the antihypertensive and lipid-lowering treatment to prevent heart attack trial. J Clin Hypertens (Greenwich) 2014 May;16(5):323-330.

33. Hoshino A, Nakamura T, Matsubara $\mathrm{H}$. The bedtime administration ameliorates blood pressure variability and reduces urinary albumin excretion in amlodipine-olmesartan combination therapy. Clin Exp Hypertens 2010 Nov;32(7):416-422.

34. Levi-Marpillat N, Macquin-Mavier I, Tropeano AI, Parati G, Maison P. Antihypertensive drug classes have different effects on short-term blood pressure variability in essential hypertension. Hypertens Res 2014 Jun;37(6):585-590. 